\title{
La valutazione delle ulcere vascolari degli arti inferiori attraverso la Wound Trend Scale: l'esperienza dell'Azienda USL di Piacenza Assessment of vascular ulcers of the lower limbs through the Wound Trend Scale: the experience of the Local Health Authority of Piacenza
}

\author{
Fabio Mozzarelli, Sara Gaetti
}

Reparto di Chirurgia Generale e Vascolare, Ospedale Guglielmo Da Saliceto, Piacenza, Italia

\section{RIASSUNTO}

Le ulcere cutanee vascolari degli arti inferiori, siano esse di origine venosa, arteriosa o mista, rappresentano una patologia diffusa che colpisce dallo 0,3 al 5\% della popolazione, consumando fra l'1 ed il 3\% del fondo sanitario Nazionale. La cura delle persone con questo tipo di lesioni deve avvalersi di strumenti idonei alla valutazione delle caratteristiche delle ulcere in modo che possa innescare il trattamento clinico/assistenziale più adeguato. Il presente studio intende verificare l'appropriatezza nella descrizione delle lesioni e la capacità predittiva sull'evoluzione delle stesse della Wound Trend Scale (WTS). La ricerca è stata condotta presso l'unità operativa di chirurgia vascolare e nell'ambulatorio di vulnologia dell'Azienda USL di Piacenza; ha coinvolto 11 pazienti con 12 lesioni vascolari degli arti inferiori. Il campione di convenienza utilizzato era composto per la maggior parte da pazienti di sesso femminile (73\%) con un'età media di 79,2 anni (DS $\pm 0,7)$. È emersa una maggiore frequenza di ulcere venose (58\%) prevalentemente localizzate in sede perimalleolare (50\%). La WTS composta da 14 items, oltre ad informare rispetto alle caratteristiche delle lesioni, permette di indicare l'evoluzione delle stesse attraverso tre misurazioni seriate con intervalli stabiliti in base alla gravità della ferita. Dallo studio è emerso che l'83\% delle ulcere ha mostrato un'evoluzione verso la guarigione, mentre il restante $17 \%$ è stato connotato da un peggioramento della lesione. La WTS si pone come un valido strumento per la descrizione delle ulcere con caratteristiche di predittività rispetto all'evoluzione delle stesse ma necessita di maggiori approfondimenti per testarne l'appropriatezza.

\section{ABSTRACT}

Vascular ulcers of the lower limbs, be they venous, arterial or mixed, represent a widespread pathology affecting from 0,3 to 5\% of the population, consuming between 1 and 3\% of the National health fund. The care of people with this type of injury must use tools that are suitable for assessing the characteristics of ulcers so that it can trigger the most appropriate care treatment. The present study intends to verify the appropriateness in the description of the lesions and the predictive ability on the evolution of the same ones of the Wound Trend Scale (WTS). The research was conducted

Corrispondenza: Fabio Mozzarelli, Reparto di Chirurgia Generale e Vascolare, Ospedale Guglielmo Da Saliceto, Via G. Taverna 46, Piacenza, Italia.

Tel.: +39.339.7498072.

E-mail: fabio.mozzarelli@gmail.com

Parole chiave: ulcere vascolari, wound trend scale, predittività; vascular ulcers, wound trend scale, predictability.

Contributi: FM, supervisione e del coordinamento del lavoro; SG, raccolta e analisi dei dati.

Conflitto d'interesse: gli autori dichiarano l'assenza di conflitti d'interesse.

Fondi: nessuno.

Ricevuto per la pubblicazione: 14 dicembre 2017.

Accettato per la pubblicazione: 26 febbraio 2018.

This work is licensed under a Creative Commons Attribution NonCommercial 4.0 License (CC BY-NC 4.0).

(C) Copyright F. Mozzarelli, S. Gaetti, 2018

Licensee PAGEPress, Italy

Italian Journal of Wound Care 2018; 2(1):18-21

doi:10.4081/ijwc.2018.19 at the vascular surgery ward and in the volnology service of the Health Authority of Piacenza; involved 11 patients with 12 lower limb vascular ulcers. The convenience sample used was mostly composed of female patients (73\%) with a mean age of 79,2 years (DS \pm 0.7$)$. A greater frequency of venous ulcers (58\%) has emerged, mainly located in the perimalleolar site (50\%). The WTS consists of 14 items, in addition to informing the characteristics of the lesions, premise to indicate the evolution of the same through three measurements with intervals established based on the severity of the wound. The study found that $83 \%$ of ulcers showed an evolution towards healing, while the remaining $17 \%$ was characterized by a worsening of the lesion. The WTS stands as a valid tool for the description of ulcers with predictive characteristics with respect to their evolution but needs more in-depth analysis to test their appropriateness.

\section{INTRODUZIONE}

La problematica delle lesioni cutanee da insufficienza circolatoria degli arti inferiori, colpisce un rilevante numero di persone stimate fra lo $0,3 \%$ e il $5 \%$ della popolazione generale, ${ }^{1}$ con una prevalenza del 3,6\% nei soggetti oltre i 65 anni. $^{2}$ 
Gli aspetti clinici e sociali della patologia sono spesso affrontati dai familiari del paziente e dalla rete sociale informale influendo negativamente sulla qualità della vita del soggetto e dal suo entourage.

Nondimeno, il peso economico del fenomeno si attesta fra 1 ' $1 \%$ e il 3\% del budget sanitario nazionale rispettivamente in Inghilterra, in Italia e in Svezia. ${ }^{3-5}$

L'utilizzo delle risorse per questo gruppo di pazienti è ripartito fra l'impegno del personale infermieristico, l'uso di dispositivi e medicazioni avanzate e il ricovero ospedaliero necessario alla effettuazione di accertamenti diagnostici e trattamenti clinici sistemici e topici. ${ }^{6}$

Le ulcere di nuova insorgenza e anche quelle croniche impongono lunghi tempi di trattamento; infatti, si stima che il range temporale per la guarigione di una nuova lesione sia di 80 giorni mentre per quelle croniche si attesti a 117 giorni. $^{7}$

La presa in cura delle persone con tali problematiche deve primariamente considerare gli aspetti clinici e assistenziali ${ }^{8,9}$ ma deve anche contemplare una congrua modalità valutativa avvalendosi di strumenti che possano descrivere, con criteri oggettivi e riconosciuti, le caratteristiche delle lesioni ulcerose degli arti inferiori.

Il lavoro di ricerca riportato intende indagare quanto la wound trend scale (WTS ${ }^{10}$ possa informare sulle peculiarità di tali lesioni e se esprime qualità predittive rispetto alla guarigione o al peggioramento delle ulcere di origine vascolare.

\section{MATERIALI E METODI}

La Wound Trend Scale è uno strumento che prevede la valutazione delle lesioni vascolari attraverso tre rilevazioni consecutive temporizzate in base allo stato della lesione.

La programmazione consiste in un assessment ogni dieci giorni se l'ulcera presenta un elevato rischio di non guarigione o di amputazione oppure ogni quindici giorni se il rischio è moderato e una valutazione al mese quando la lesione presenta indici di stabilità.

La WTS è composta da 14 parametri, quali le dimensioni dell'ulcere (lunghezza $\times$ larghezza), la profondità, il margine della lesione, il bordo sotto minato, la presenza di fistola o cavità ascessuale, la percentuale del margine della ferita sotto minato, il tipo di tessuto di base della ferita, la presenza e la quantità di essudato, le caratteristiche della cute perilesionale, l'evidenza di edema, di indurimento, di granulazione, di epitelizzazione e il rischio di infezione. Essa può totalizzare un punteggio minimo di 12 punti fino al un massimo di 58 punti (e oltre).

A ogni osservazione, l'infermiere riporta un valore per ciascun parametro e il riscontro di una somma inferiore rispetto alla valutazione precedente viene interpretata come una modifica positiva che può essere probante per un miglioramento della ferita. All'opposto, un aumento di tali valori può invece essere indice di un deterioramento.

La domanda di ricerca si è concentrata sula capacità della WTS di fornire informazioni circa

l'evoluzione della lesione vascolare applicandola per tre volte consecutive.

Lo studio si è svolto presso l'unità operativa di Chirurgia Vascolare e negli ambulatori di vulnologia dell'Azienda USL di Piacenza. Il periodo di studio si è sviluppato fra aprile e settembre dell'anno 2017 selezionando un campione consecutivo di convenienza, includendo tutti i pazienti che presentano ulcere vascolari venose, arteriose e miste. Sono stati esclusi i pazienti che presentavano ulcere da decubito, diabetiche, neoplastiche e lesioni da deiscenza chirurgica.

\section{RISULTATI}

I dati raccolti hanno interessato un campione formato da 11 pazienti ( 8 femmine $\mathrm{e} 3$ maschi) con un'età media di 79,2 anni (DS $\pm 0,7$; Range 75-84 anni) che presentavano 12 ulcere vascolari. In merito alle tipologie di lesioni emerse, 7 erano di tipo venoso, 3 di tipo arterioso e 2 miste (Figura 1).

Le sedi degli arti inferiori colpite dalle ulcere vascolari osservate durante lo studio erano quella perimalleolare $(50 \%)$, tibiale $(33,3 \%)$, e dorso del piede $(16,7 \%)$ (Figura 2).

La misurazione seriata per tre volte attraverso la WTS ha evidenziato che $10(83 \%)$ lesioni cutanee hanno presentano un'evoluzione verso la guarigione.

In merito alle ulcere che hanno mostrato una progressione peggiorativa, si nota che vi è una maggiore frequenza nelle persone di sesso femminile con lesioni in sede malleolare (Figura 3). Per quanto concerne le ferite migliorate, si evince una maggiore numerosità nel sesso femminile, con ulcere di tipo venoso che si presentano maggiormente in sede tibiale (Figura 4).

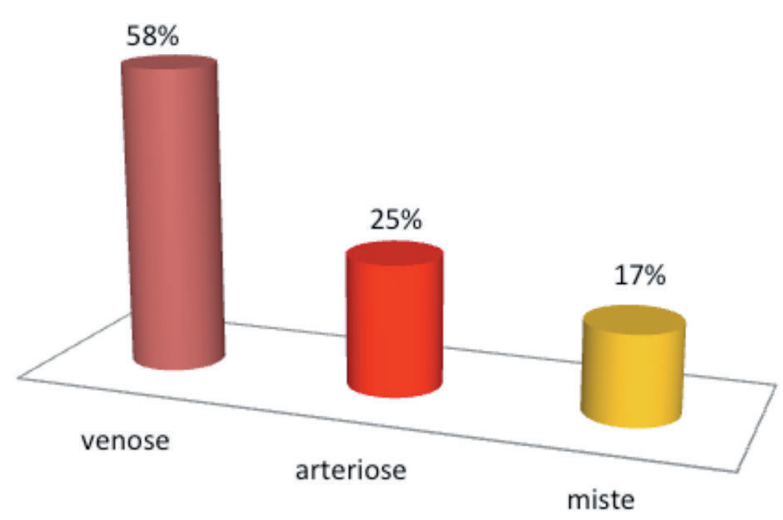

Figura 1. Tipologie di ulcere vascolari. 


\section{DISCUSSIONE}

Elaborando le informazioni ottenute, deduciamo che attraverso l'applicazione della scala di valutazione WTS, sia possibile descrivere e monitorare l'evoluzione dell'ulcera del paziente verso la guarigione o il deterioramento.

Dai risultati emerge che i soggetti maggiormente predisposti a sviluppare ulcere vascolari agli arti inferiori siano di sesso femminile; sono prevalentemente di natura venosa e la sede in cui si instaurano con più frequenza risulta essere quella perimalleolare.

Mediante l'applicazione della scala di valutazione WTS su un totale di 11 pazienti e di 12 ulcere vascolari, si è osservato che in cinque casi vi è stata una diminuzione del punteggio sia alla prima che alla seconda misurazione ed anche fra la seconda e la terza.

Tre ulcere hanno mantenuto un punteggio uguale in tutte le valutazioni mentre una di queste ha riportato un aumento del punteggio totale durante l'ultima applicazione della scala.

In due situazioni, si è osservata un'evoluzione verso il deterioramento (17\%) e 10 verso la guarigione (83\%).

Nella scala WTS sono presenti tutti i parametri necessari per un'analisi chiara e completa dell'ulcera vascolare; essi sono ampiamente descritti dalle linee guida per la gestione delle tali lesioni e rappresentati dall'acronimo TIME (tissue, infection, moisture, edge) ${ }^{11}$ ovvero la valutazione del tessuto, la presenza di segni di infezione, le caratteristiche dei fluidi presenti sulla ferita e le caratteristiche dei margini della lesione.

L'utilizzo di uno strumento completo come la WTS può garantire non solo una valutazione approfondita dell'ulcera vascolare, ma è anche in grado di fornire un linguaggio comune per i professionisti dedicati al loro trattamento. Una stima appropriata della lesione favorisce lo sviluppo di una diagnosi adeguata e la stesura di una pianificazione specifica per i singoli individui. La WTS può essere intesa anche come una guida che concorre a programmare il trattamento opportuno e risolutivo, favorendo un'idonea presa in carico del paziente al fine di ridurre gli innumerevoli rischi e le molteplici complicanze a cui sono esposti i soggetti che presentano lesioni vascolari. La suddetta scala, a confronto di altri dispositivi, è ideata esclusivamente per le lesioni cutanee degli arti inferiori e considera alcuni parametri quali l'edema, il rischio d'infezione e il dolore che non vengono presi in considerazione da altri strumenti come ad esempio la Push tool. ${ }^{12}$

Secondo i risultati di letteratura, la WTS esprime un indice di specificità del $87 \%$ e di sensibilità del $99 \%$ con un valore predittivo positivo del $96 \%{ }^{10}$

Dallo studio presentato emergono alcuni limiti quali le piccole dimensioni del campione che possono aver influito negativamente sullo svolgimento dell'indagine indebolendo i risultati ottenuti mentre per quanto attiene agli

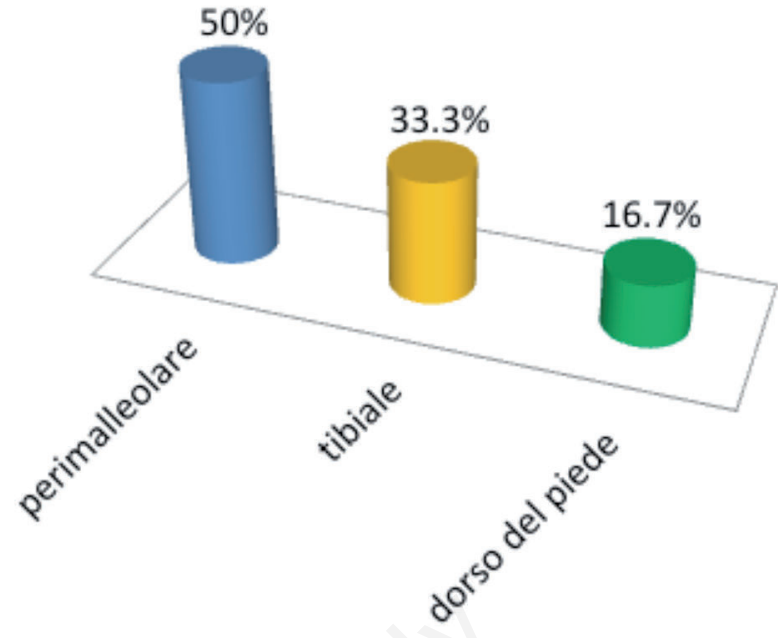

Figura 2. Sede anatomica delle ulcere vascolari.

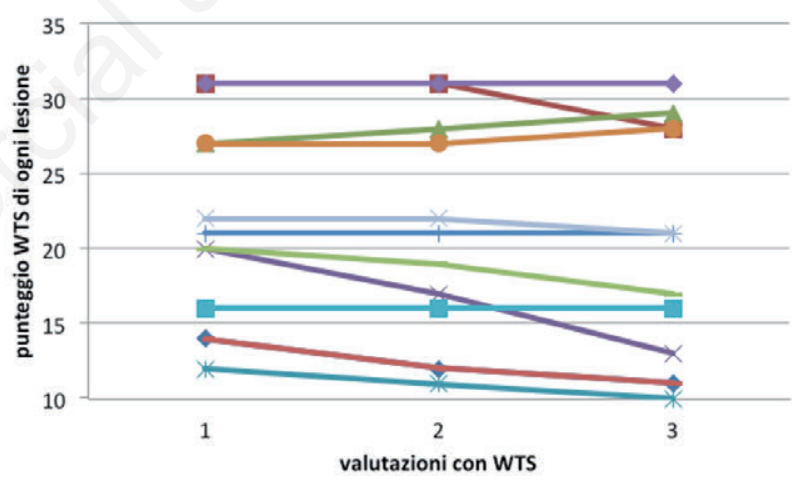

Figura 3. Esito delle ulcere vascolari a progressione peggiorativa.

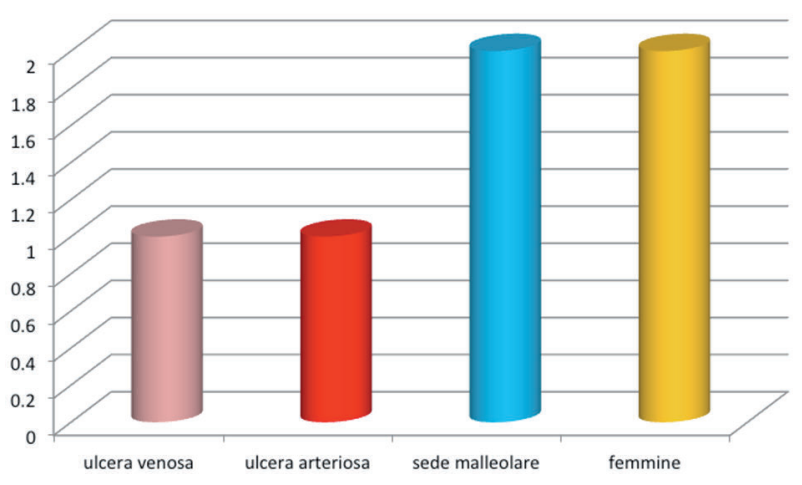

Figura 4. Ulcere vascolari con progressione migliorativa. 
aspetti positivi, possono essere riportati la modalità di raccolta dei dati che è stata effettuata da un unico professionista riducendo eventuali difetti dovuti alla interpretazione degli items proposti dalla WTS e che il trattamento locale e sistemico delle lesioni in studio veniva sempre effettuato dallo stesso team di cura.

\section{CONCLUSIONI}

Le ulcere vascolari agli arti inferiori rappresentano un problema di salute estremamente comune che ha un'influenza importante sia dal punto di vista epidemiologico che socio-economico. Diviene necessario introdurre all'interno della pratica clinica, uno strumento di valutazione che possa essere specifico, rapido, di facile utilizzo e che permetta di individuare precocemente $\mathrm{i}$ cambiamenti sia in senso peggiorativo che migliorativo delle lesioni cutanee della persona che prendiamo in cura. La scala di valutazione Wound Trend Scale è uno strumento dinamico ed efficace che racchiude tutti i parametri necessari per un inquadramento diagnostico dettagliato. $\mathrm{Ci}$ consente di descrivere ciò che osserviamo, di delineare l'andamento della patologia e ci suggerisce un linguaggio comune. La WTS si pone come strumento idoneo agli scopi descritti ma necessita di analisi più rigorose per testarne l'appropriatezza.

\section{BIBLIOGRAFIA}

1. Morison M, Moffatt C, Franks P. Leg ulcers: a problembased learning approach. USA: Mosby; 2007.

2. Marazzi M. Le ulcere cutanee: l'importanza dell'approccio preparatorio della ferita per raggiungere la guarigione. Il debridement. Riv Soc It Med Gen 2010. Disponibile all'indi- rizzo: https://www.simg.it/Documenti/Rivista/2010/06 2010/5.pdf. Consultato ad agosto 2017.

3. Wilson E. Prevention and treatment of venous leg ulcers. Health Trends 1989:21:97.

4. Appelquist J, Ragnarson-Tennvall G, Persson U, Larsson J. Diabetic foot ulcers in a multi-disciplinary setting. An economic analysis of primary healing with amputation. J Int Med 1994;235:463-71.

5. Nelzén O, Bergqvist D, Lindhagen A. Venous and non-venous leg ulcers. Br J Surg 1994;81:182-7.

6. Marklund B, Bengtsson C, Bryntesson P, et al. Telephone advisory service, visits to district nurses and home visits made by district nurses at a Swedish primary health care district. Scand J Prim Health Care 1991;9:161-6.

7. Lévy E, Lévy P. Management of venous leg ulcer by French physicians, diversity and related costs: a prospective medioeconomic osservational study. J Mal Vasc. 2001;26:39-44.

8 . Royal college of nursing. The nursing management of patients whit venous leg ulcers: audit protocol. Disponibile all'indirizzo: https://www.rcn.org.uk/professional-development/publications/pub-001269. Consultato a maggio 2017.

9. European Wound Management Association. Management of patients whit venous leg ulcers. Disponibile all'indirizzo: http://www.aiuc.it/files/EWMAvenouslegulcers.pdf. Consultato a maggio 2017.

10. Noreen A, Campbell RN, Donna L, et al. Ostomy Wound Management. The Wound Trend Scale: a retrospective review of utility and predictive value in the assessment and documentation of lower leg ulcers. Disponibile all'indirizzo: http://www.o-wm.com/article/wound-trend-scale-retrospective-review-utility-and-predictive-value-assessment. Consultato a giugno 2017.

11. Associazione Italiana Ulcere Cutanee Onlus. I principi del TIME per la valutazione della ferita. Disponibile all'indirizzo: http://www.aiuc.it/pagina/383/i+principi+del+time+ per+la+valutazione+della+ferita. Consultato a giugno 2017.

12. Ratliff CR, Rodeheaver GT. Use of the PUSH tool to measure venous ulcer. Ostomy/Wound Manag 2005;51:58-63. 